\title{
Quantum sensors for microscopic tunneling systems
}

\author{
Alexander Bilmes $\mathbb{D}^{1 凶}$, Serhii Volosheniuk $\mathbb{D}^{1}$, Jan David Brehm $\mathbb{D}^{1}$, Alexey V. Ustinov ${ }^{1,2,3}$ and Jürgen Lisenfeld $\mathbb{D}^{1}$
}

The anomalous low-temperature properties of glasses arise from intrinsic excitable entities, so-called tunneling Two-Level-Systems (TLS), whose microscopic nature has been baffling solid-state physicists for decades. TLS have become particularly important for micro-fabricated quantum devices such as superconducting qubits, where they are a major source of decoherence. Here, we present a method to characterize individual TLS in virtually arbitrary materials deposited as thin films. The material is used as the dielectric in a capacitor that shunts the Josephson junction of a superconducting qubit. In such a hybrid quantum system the qubit serves as an interface to detect and control individual TLS. We demonstrate spectroscopic measurements of TLS resonances, evaluate their coupling to applied strain and DC-electric fields, and find evidence of strong interaction between coherent TLS in the sample material. Our approach opens avenues for quantum material spectroscopy to investigate the structure of tunneling defects and to develop low-loss dielectrics that are urgently required for the advancement of superconducting quantum computers.

npj Quantum Information (2021)7:27; https://doi.org/10.1038/s41534-020-00359-x

\section{INTRODUCTION}

We are still lacking an explanation for the behaviour of amorphous materials at low temperatures $<10 \mathrm{~K}^{1,2}$. Why is it that even widely different materials ranging from biatomic glasses to polymers show quantitatively identical properties such as specific heat and thermal conductivity ${ }^{3}$ ? The Standard Tunneling Model (STM) ${ }^{4,5}$ has been a first attempt to explain these universal anomalies on the basis of two-level systems (TLS) believed to arise from the tunneling of atoms between two energetically similar configurations in the disordered lattice structure. While the STM neglects mutual TLS interactions and fails in the intermediate temperature range of $1-10 \mathrm{~K}$, refined models include TLS-TLS interactions ${ }^{6-8}$, assume different types of TLS ${ }^{9}$, or consider specific dependencies of TLS potential energies ${ }^{10,11}$. Since insights from experiments on bulk materials were limited to observing the averaged response from large and inhomogeneous ensembles of TLS, their individual properties remained out of reach.

This situation has changed with the advent of superconducting qubits that realize well-controllable macroscopic quantum systems with custom-tailored energy spectra and couplings to the environment. Qubits are implemented from electric resonant circuits employing Josephson tunnel junctions that serve as nonlinear inductances to obtain anharmonic potential wells where discrete eigenstates can be selectively addressed. Driven by the desire to realize solid-state quantum information processors, intensive effort went into the development of advanced circuit designs $^{12-16}$ and fabrication techniques ${ }^{17,18}$, which resulted in a dramatic improvement of device coherence. The entry of commercial enterprises has further accelerated progress, culminating in the demonstration of machine learning algorithms ${ }^{19}$, access to prototype quantum processors via the $\operatorname{cloud}^{20}$, and the achievement of quantum supremacy by controlling a 53-qubit system that could not anymore be simulated efficiently by classical supercomputers ${ }^{21}$.

Despite these achievements, progress towards truly large-scale quantum processors is still hindered by decoherence, of which the major part is due to losses in dielectric circuit materials ${ }^{22}$. TLS residing in the tunnel barriers of Josephson junctions and in the native surface oxides of superconducting electrodes may couple via their electric dipole moments to the qubit's oscillating E-field. When TLS are at resonance with the qubit, they can efficiently dissipate energy into the phonon ${ }^{23}$ or BCS-quasiparticle ${ }^{24}$ bath which results in reduced qubit energy relaxation times $T_{1}{ }^{25}$ and, in the case of strong coupling, gives rise to avoided level crossings in qubit spectroscopy ${ }^{26}$. Moreover, thermally activated TLS at low energies may interact with high-energy TLS that have frequencies near resonance with a qubit or resonator, and this causes temporal fluctuations of the device's resonance frequency ${ }^{27,28}$ and energy relaxation rate re-32. $^{29}$.

Further progress with superconducting quantum processors based on current circuit architectures thus requires extensive material and fabrication process research to avoid the formation of TLS. Moreover, tools to verify the quality of metal films and junctions are required that are able to relate fabrication processes to TLS formation and to investigate the microscopic nature of the material defects. For these tasks, qubits themselves are well suited because of their sensitivity to TLS. In case of strong coupling, quantum state swapping between the qubit and $\operatorname{TLS}^{33}$ is possible, allowing one to characterize TLS' coherence properties ${ }^{23,34,35}$, and their coupling to the environment ${ }^{24,36,37}$. A useful method for such studies is to control the TLS' internal asymmetry energy and thus their resonance frequency by applied mechanical strain ${ }^{38}$ or DCelectric field ${ }^{39}$. Operating qubits in electric fields allows one to distinguish defects in tunnel junction barriers from those on electrode surfaces ${ }^{40}$ and to obtain information on the positions of individual TLS in the quantum circuit ${ }^{41}$.

In this letter, we present a quantum sensor that grants access to measurement and manipulation of individual TLS in virtually arbitrary materials. The device is based on a transmon qubit ${ }^{13,25}$, which consists of a capacitively shunted DC-SQUID formed by two Josephson junctions connected in parallel, as shown in Fig. 1a, c. Qubit readout is performed by measuring the dispersive resonance frequency shift of a coplanar resonator that is capacitively coupled to the qubit. The qubit resonance frequency can be tuned in a range of typically $\approx 1 \mathrm{GHz}$ by an on-chip coil 

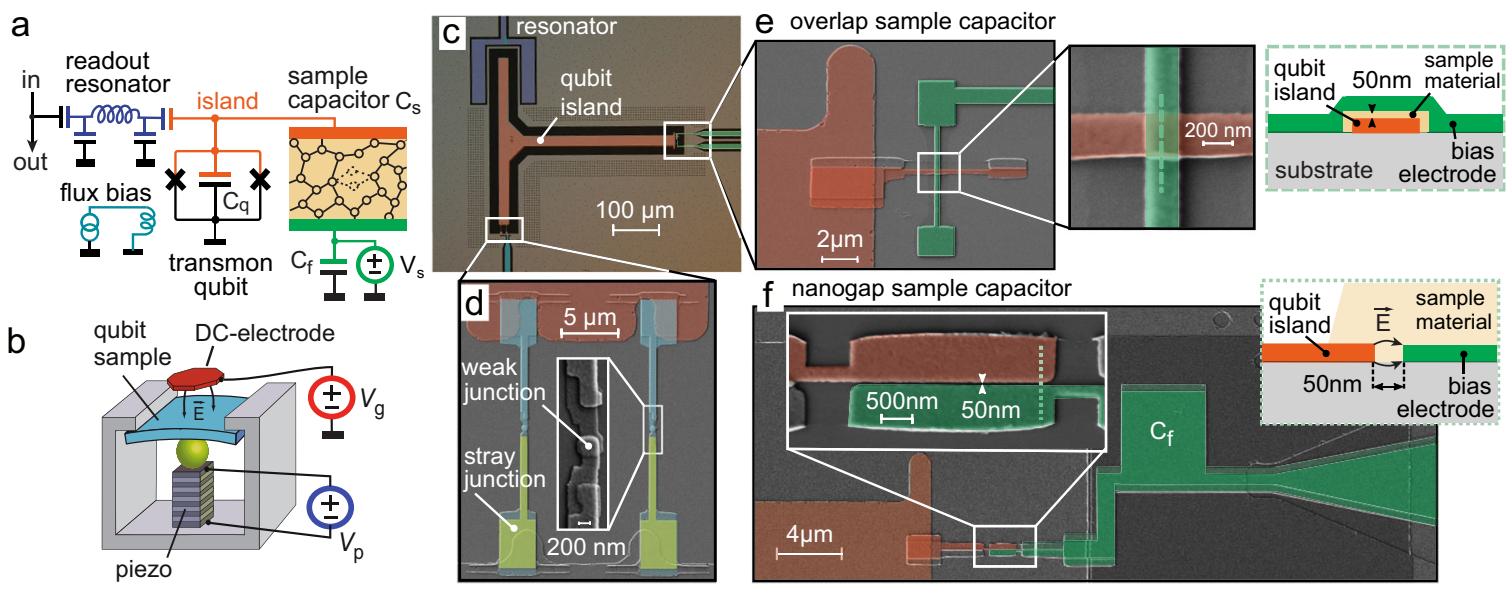

Fig. 1 Experimental setup and qubit sample. a Schematic of the transmon qubit circuit to study TLS in deposited materials. The qubit island (red) is connected to ground by an additional small capacitor containing the material to be studied. b Setup for tuning TLS by applied mechanical strain and a global DC-electric field. $\mathbf{c}$ Photograph of the qubit island. $\mathbf{d}$ DC-SQUID connecting the qubit island to ground, and a zoom onto one of the small Josephson junctions. The large-area stray junctions are highlighted in light green. e Sample capacitor in overlap geometry as used in this work. It employs a 50-nm thick layer of $\mathrm{AlO}_{x}$ as the sample dielectric. $\mathbf{f}$ An alternative sample capacitor design consists of two coplanar electrodes separated by a so-called 'nanogap' of a few tens of $\mathrm{nm}$. Here, the sample material can be deposited in a last fabrication step (see inset), or the nanogap can be left uncovered to study individual TLS in native surface oxides.

providing magnetic flux, which frustrates the Josephson energy of the DC-SQUID loop shown in Fig. 1d.

The material under test defines the dielectric in an additional "sample capacitor" shunting the transmon qubit. In this work, we use a capacitor having a plate or "overlap" geometry as shown in Fig. 1e. This allows one to study TLS in all dielectrics that can be deposited as thin films, e.g., by sputtering or evaporation. Alternatively, one can employ a so-called nanogap capacitor consisting of two coplanar electrodes (see Fig. 1f) that are separated by a few tens of nanometres, and then covered by the sample material. In this case, the coupling between TLS and the qubit occurs via the fringing electric field sketched in the inset of Fig. $1 \mathrm{f}$. This provides the possibility to study TLS in pieces of bulk material by pressing it onto the nanogap capacitor. Moreover, the use of uncovered nanogap capacitors allows one to study single TLS residing in the native oxides of the electrode material and defects that are formed by surface adsorbates.

The STM describes TLS on the basis of a double-well potential whose minima differ by the asymmetry energy $\varepsilon$, and transitions between wells occur at a tunneling energy $\Delta_{0}$, resulting in the transition energy $E=\sqrt{\Delta_{0}^{2}+\varepsilon^{2}}$. TLS in the sample material couple to the qubit at a strength $\hbar g=\mathbf{p F}=\bar{p}|\mathbf{F}|$, where $\mathbf{F}$ is the electric field inside the capacitor, which is induced by the qubit plasma oscillation, and $\bar{p}=p_{\|}\left(\Delta_{0} / E\right)$ is the projection of the TLS' dipole moment $\mathbf{p}$ onto $\mathbf{F}$, multiplied by the TLS' matrix element ${ }^{39} \Delta_{0} / E$.

\section{RESULTS}

\section{Sample capacitor design}

Single TLS can be detected if their energy exchange rate with the qubit (which equals their coupling strength $g$ at resonance) is comparable to the energy decay rate $1 / T_{1}$ of the isolated qubit. The criterium $g \approx 1 / T_{1}$ togeher with the TLS' above-mentioned coupling strength $\hbar g=\bar{p}|\mathbf{F}|$ define a suitable thickness $d$ of the dielectric layer in overlap capacitors: $d=\bar{p} T_{1} V_{\text {rms }} / \hbar$, where the electric field $|\mathbf{F}|$ is substituted by $V_{\text {rms }} / d$. Here, $V_{\text {rms }}=\sqrt{\hbar \omega_{10} / 2 C_{\text {tot }}} \approx 4.5 \mu \mathrm{V}$ is the vacuum voltage fluctuation on the qubit island at the designed plasma oscillation frequency $\omega_{10} \approx 2 \pi \cdot 6.2 \mathrm{GHz}$ when $C_{\text {tot }} \approx 100 \mathrm{fF}$ is the sum of all capacitances shunting the qubit. To be able to detect a TLS dipole moment $p_{\|}$of minimum $0.1 e \AA^{42}$, and assuming a rather conservative $T_{1} \approx 1 \mu \mathrm{s}$, we arrive at a dielectric layer thickness $d \approx 70 \mathrm{~nm}$. We chose $d=50 \mathrm{~nm}$ and a capacitor size of $(0.25 \times 0.3)$ $\mu \mathrm{m}^{2}$, resulting in $C_{\mathrm{s}} \approx 0.15 \mathrm{fF} \ll C_{\text {tot }}$ which ensures that the energy that is stored in the lossy sample capacitor remains limited to a small fraction of the qubit's total energy, and coherence is preserved. A picture of the employed sample capacitor is shown in Fig. 1e while further details on the capacitor design are given in Supplementary Methods 1.

It is furthermore necessary to be able to distinguish TLS in the sample material from those on electrode interfaces and from TLS in Josephson junctions. This is accomplished by probing the TLS' response to a local electric field generated by voltage-biasing the sample capacitor's electrode as indicated in Fig. 1a, where the additional capacitor $C_{\mathrm{f}} \sim 250 \mathrm{fF}$ serves as a DC-break. The bias voltage will not induce an electric field in the transmon's shunt capacitor $C_{q}$ nor inside the Josephson junctions' tunnel barrier since the DC-electric potential difference of the transmon island and ground will be compensated by Cooper-pair tunneling ${ }^{40}$, so that only TLS in the sample capacitor respond to the applied voltage $V_{s}$. In addition, we can tune TLS residing at the perimeter of the qubit capacitor by a globally applied DC-electric field that is generated by an electrode installed above the qubit chip ${ }^{40}$ as shown in Fig. 1b. Moreover, all TLS including those residing inside the tunnel barriers of junctions can be tuned via physical strain by bending the chip with a piezo actuator ${ }^{38,43}$, which is useful to enhance the number of observable TLS. The table in Fig. $2 b$ summarizes how to identify the location of a TLS from its tunability characteristics.

We chose amorphous aluminum oxide $\mathrm{AlO}_{x}$ as the sample material for this work since it is well characterized and of general importance for superconducting quantum circuits where it is ubiquitously used as a reliable tunnel barrier material. The sample capacitor is patterned with electron-beam lithography, where the bottom electrode is deposited and connected to the qubit island in the same step as the qubit's Josephson junctions, followed by a third lithography step depositing $50 \mathrm{~nm}$ of $\mathrm{AlO}_{x}$ by eBeamevaporation of $\mathrm{Al}$ in an oxygen atmosphere, and capping it by a top $\mathrm{Al}$ electrode. The filter capacitor $C_{\mathrm{f}}$ is formed simultaneously as a wider section in the top electrode. Here, we report results for samples employing small sample capacitors of size $(0.25 \times 0.3) \mu \mathrm{m}^{2}$ which did not contribute significantly to decoherence. Two tested $C_{s}$-shunted qubits reached $T_{1}$-times of $3.3-4.2 \mu \mathrm{s}$, which is comparable with an isolated reference qubit $\left(T_{1} \approx 4.3 \mu \mathrm{s}\right)$ on the same chip. In another batch, we also tested larger sample 


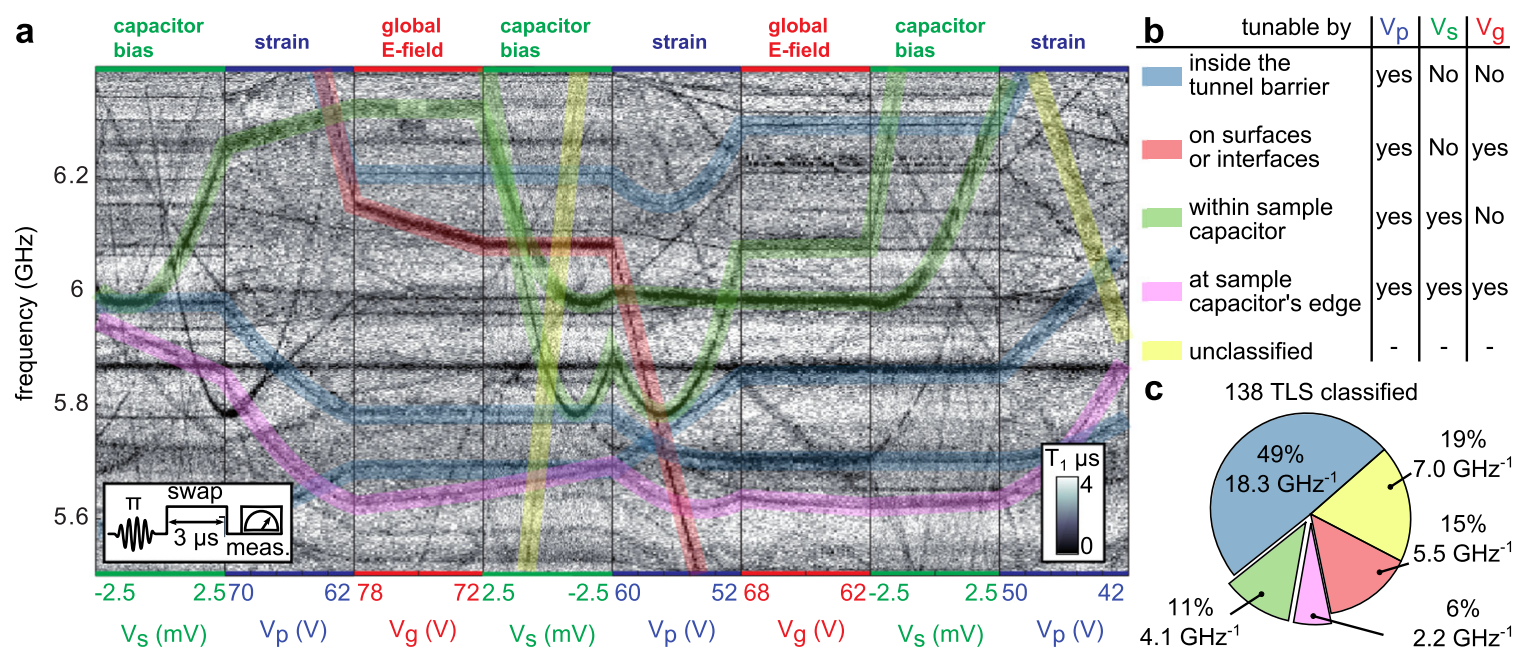

Fig. 2 Defect spectroscopy. a Typical data recorded with the swap spectroscopy protocol (see left inset). Each dark trace indicates a reduction in the qubit's $T_{1}$ time due to resonant coupling to an individual TLS. The response of TLS to control parameters (such as physical strain, global E-field, and the E-field inside the sample capacitor) allows one to identify the possible location of the TLS as listed in the table in panel $\mathbf{b}$. Some exemplary TLS are highlighted with colored lines, where yellow lines indicate TLS that could not be classified since they were visible in only a single segment. While $V_{\mathrm{g}}$ and $V_{\mathrm{p}}$ were continually reduced, $V_{\mathrm{s}}$ was ramped alternatingly up or down with the amplitude limited to $\left|V_{\mathrm{s}}\right|<2.5 \mathrm{mV}$ to avoid heating of the attenuators in the bias line. c The resulting density (detected TLS per GHz bandwidth) of 138 TLS observed in measurements on two identical qubits.

capacitors $(0.3 \times 2.1) \mu \mathrm{m}^{2}$, which did limit the qubit's $T_{1}$ time $^{44}$. This allowed us to measure the loss tangent of the employed $\mathrm{AlO}_{x}$ dielectric as $\tan \delta_{0} \approx(1.7 \pm 0.2) \cdot 10^{-3}$, comparable with other reports $^{42,45-47}$.

\section{TLS spectroscopy}

To distinguish whether a TLS is located in a tunnel barrier, at the qubit's film edges ${ }^{41}$, or in the sample capacitor dielectric, we track its resonance frequency for a range of voltages applied to the global DC electrode $\left(V_{\mathrm{g}}\right)$, to the sample dielectric $\left(V_{\mathrm{s}}\right)$, and to the piezo $\left(V_{\mathrm{p}}\right)$. An example of such a measurement is presented in Fig. 2a, showing the frequency dependence of the qubit's $T_{1}$ time estimated by swap spectroscopy ${ }^{33,35,43}$, where dark traces reveal enhanced qubit energy relaxation due to resonant TLS. These segmented hyperbolic traces are fitted to obtain the TLS' coupling constants $\gamma$ which determine their bias-dependent asymmetry energy $\varepsilon=\varepsilon_{\mathrm{i}}+\gamma_{\mathrm{g}} V_{\mathrm{g}}+\gamma_{\mathrm{s}} V_{\mathrm{s}}+\gamma_{\mathrm{p}} V_{\mathrm{p}}$ up to an intrinsic offset $\varepsilon_{\mathrm{i}}$. The fit also results in the value of the TLS' tunneling energy $\Delta_{0}$ if it lies within the tunability range of the qubit's resonance frequency.

Thanks to the well-specified DC-electric field $V_{s} / d$ in the sample capacitor, the coupling electric dipole moment $p_{\|}=\gamma_{\mathrm{s}} d / 2$ of TLS in the sample material is directly obtained from the identity $2 p_{\|} V_{s} / d=\gamma_{s} V_{s}^{4,5}$. In contrast, a measurement of the TLS' coupling strength to a quantum circuit results in the effective dipole moment size $\bar{p}$ where the matrix element $\left(\Delta_{0} / E\right)$ is often unknown. From measurements on two identical qubits in one cool-down, we characterized in total 138 TLS. Of those, 13 TLS were found inside the sample material, with a spectral density of $4.1 \mathrm{GHz}^{-1}$ (see calculation details in Supplementary Methods 3), which results in a volume density of $P_{0}=4.1\left(V_{\mathrm{d}} \mathrm{GHz}\right)^{-1}=1800\left(\mu \mathrm{m}^{3} \cdot \mathrm{GHz}\right)^{-1}$. We estimated the field-free dielectric volume $V_{d}=(0.15 \times 0.3 \times 0.05)$ $\mu \mathrm{m}^{3}$ by assuming that the global field penetrates the sample dielectric to a depth of about its thickness $(50 \mathrm{~nm})$ from the sides open to air.

\section{DISCUSSION}

The average dipole moment of the observed sample-TLS was $p_{\|}=$

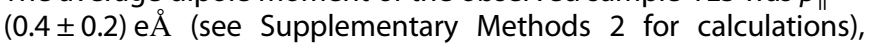

which results in a loss tangent ${ }^{48}$ of the employed $\mathrm{AlO}_{x}\left(\varepsilon_{r} \approx 10\right)$ of $\tan \delta_{0}=\pi P_{0} p_{\|}^{2}\left(3 \varepsilon_{0} \varepsilon_{r}\right)^{-1} \approx 10^{-3}$, comparable with the number quoted above. The statistics shown in Fig. $2 c$ indicate that the qubits were mostly limited by TLS hosted inside the tunnel barrier of the stray Josephson junctions (light green in Fig. 1d), which are a fabrication artefact that could have been avoided by shorting them in an additional lithography step ${ }^{49,50}$.

For the $1.5-2 \mathrm{~nm}$ thin ${ }^{51-53}$ and $17.17 \mu \mathrm{m}^{2}$ large tunnel barriers of the two stray junctions shown in Fig. 1d, our measurements indicate a TLS volume density of $P_{0, J}=360$ to $270\left(\mu \mathrm{m}^{3} \cdot \mathrm{GHz}\right)^{-1}$, in good agreement to previous work ${ }^{40}$. Notably, this is about six times smaller than the TLS density found in the thicker layer of deposited AlOx in the sample capacitor. This is probably due to the minimum detectable TLS dipole moment size, i.e., qubit's sensitivity, which is smaller for sample-TLS due to stronger oscillating qubit fields $\left(\approx 90 \mathrm{Vm}^{-1}\right)$ inside the sample capacitor, compared to the field inside the tunnel barrier of the stray junctions $\left(\approx 15 \mathrm{Vm}^{-1}\right)$. We speculate that this notion might be dressed due to various reasons like a reduced dangling bond density due to facilitated atom diffusibility and self-annealing in the thin tunnel barrier ${ }^{54}$, or enhanced shielding of TLS by the evanescent Cooper-pair condensate ${ }^{44}$, or reasons related to the material's different growth conditions.

E-field spectroscopy also revealed coherent mutual interactions between TLS in the sample material, which manifest themselves in avoided level crossings as shown in Fig. 3. The coupling between the TLS is described by the interaction Hamiltonian $H_{\text {int }}=\frac{\hbar}{2}\left(g_{x} \sigma_{1}^{x} \sigma_{2}^{x}+g_{z} \sigma_{1}^{z} \sigma_{2}^{z}\right)$, where $\sigma_{i}^{x}$ and $\sigma_{i}^{z}$ are the Pauli matrices of TLS $i$. As an advancement over earlier work ${ }^{43}$, the combined control of strain and local E-field allowed us to mutually detune the TLS and shift the avoided level crossing through the symmetry point of the observed TLS as demonstrated by the lower panels of Fig. 3. Since the longitudinal coupling component $g_{z} \propto \sigma_{1}^{z}$ changes its sign when TLS 1 is tuned through its symmetry point, its effect can be well distinguished from the transversal component $g_{x}$. This enabled fitting of both components $g_{x}=-19(\mu \mathrm{s})^{-1}$ and $g_{z}=25(\mu \mathrm{s})^{-1}$. More details on the description of coherently interacting TLS can be found in a previous work ${ }^{43}$ and in Supplementary Methods 4. 

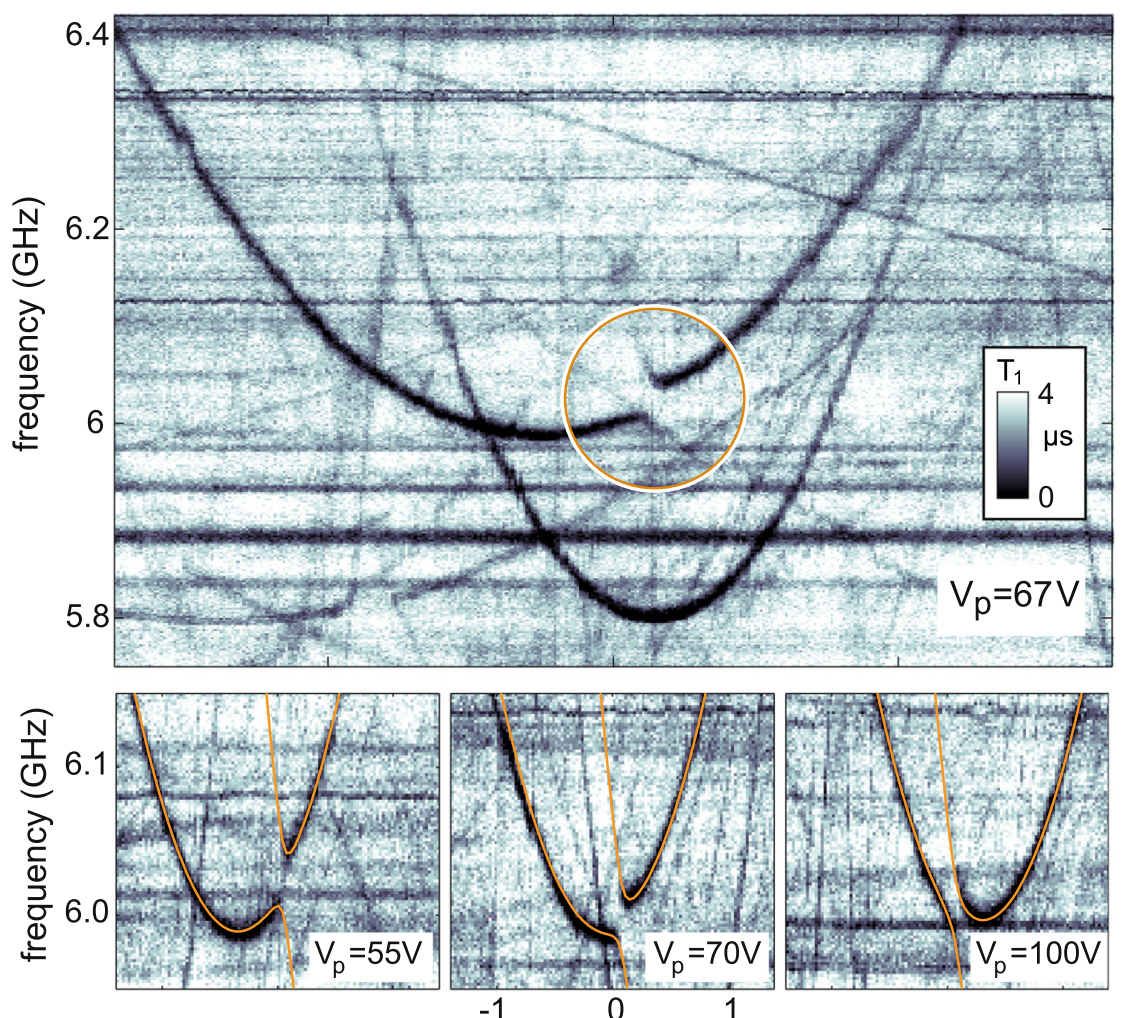

capacitor bias voltage $\mathrm{V}_{\mathrm{S}}(\mathrm{mV})$

Fig. 3 Interacting TLS in the sample dielectric. Top: Avoided level crossing (encircled) in the spectrum of a TLS due to coherent interaction with a second TLS. Bottom: The observed level splitting could be shifted through the TLS symmetry point by mutually detuning the two TLS via the physical strain. Each figure was recorded for the same range of bias voltages but at different voltage $V_{p}$ applied at the piezo actuator. Superimposed orange lines show the transition frequencies calculated using independently measured TLS parameters and best-fitting interaction strengths.

In conclusion, we demonstrated that superconducting qubits can serve as interfaces for studying quantum properties of individual atomic-size tunneling systems located in arbitrary materials deposited as thin films. Qubit swap spectroscopy in dependence on the applied electric field bias to the sample material enables precise measurement of the TLS' coupling dipole moments and reveals avoided level crossings, which herald coherent interaction between TLS. The possibility to mutually detune interacting TLS by using mechanical strain as a second control parameter allows one to fully characterize the type of the interaction. The demonstrated approach has a large potential to provide further insights into the puzzling physics of amorphous solids. It may serve as a valuable tool in the search for low-loss materials urgently needed to advance nano-fabricated devices and superconducting quantum processors where TLS play a detrimental role.

\section{METHODS}

\section{Sample fabrication}

The qubit samples were fabricated and characterized at KIT. A microchip contained three independent Xmon qubits of whom two were shunted by a sample capacitor, and a third one served as a reference qubit. The qubit electrode, ground plane and resonators were patterned into a 100-nm thick Al film with an inductively coupled $\mathrm{Ar}-\mathrm{Cl}$ plasma. After Argon-ion milling ${ }^{55}$ of the optically patterned electrodes in a PLASSYS shadow evaporation device, the Josephson junctions were deposited in a subsequent electron-beam lithography step.

Qubit samples with large and small sample capacitors were studied. The bottom electrode of large sample capacitors consisted a narrow extension of the qubit island. The bottom layer of the small sample capacitor (see
Fig. 1e) was made simultaneously with the Josephson junctions. Sample dielectric and top electrodes of both capacitor types were formed in the PLASSYS device using an MMA/PMMA copolymer mask patterned in an electron-beam lithography step. After removing the native oxide of the bottom electrode with the Ar milling process, the sample dielectric (here $50 \mathrm{~nm} \mathrm{AlO}_{x}$ ) was formed during a perpendicular deposition of $\mathrm{Al}$ at a rate of $0.2 \mathrm{~nm} \mathrm{~s}^{-1}$ in an oxygen atmosphere (chamber pressure of $3 \times 10^{-4}$ $\mathrm{mBar}$, oxygen flow of $5 \mathrm{sccm}$ ). The dielectric was in situ covered by perpendicularly deposited $100-\mathrm{nm}$ thick layer of Al that formed the top electrode. Further details are reported in the PhD thesis by $A B$, Chap. 3.2.3.

\section{Experimental setup}

The sample was measured in an Oxford Kelvinox 100 wet dilution refrigerator at a temperature of $30 \mathrm{mK}$. The qubit chip was installed in a light-tight aluminium housing protected by a cryoperm magnetic shield. The coaxial control lines were heavily attenuated, filtered, and equipped with custom-made infrared filters. The qubit state was detected via the dispersive shift ${ }^{12}$ of a notch-type readout resonator, which was capacitively coupled to the qubit, and probed in a standard homodyne microwave detection setup.

The DC-gate for tuning the surface-defects consisted of a copper-foil/ Kapton foil stack that was glued to the lid of the sample box. It was connected via a twisted pair equipped with an RC-lowpass filter (cutoff ca $10 \mathrm{kHz}$ ) at the $1 \mathrm{~K}$-stage, and a custom-made copper powder lowpass filter ( $1 \mathrm{MHz}$ cutoff) at the $30 \mathrm{mK}$ stage. The top electrode of the sample capacitor was controlled via an attenuated microwave line, as further detailed in the Supplementary Methods 2.

\section{DATA AVAILABILITY}

Data are available upon reasonable request. 
Received: 8 September 2020; Accepted: 2 December 2020; Published online: 05 February 2021

\section{REFERENCES}

1. Zeller, R. C. \& Pohl, R. O. Thermal conductivity and specific heat of noncrystalline solids. Phys. Rev. B 4, 2029-2041 (1971).

2. Leggett, A. J. \& Vural, D. C. "Tunneling two-level systems" model of the lowtemperature properties of glasses: are "smoking-gun" tests possible? J. Phys. Chem. B 117, 12966-71 (2013).

3. Freeman, J. J. \& Anderson, A. C. Thermal conductivity of amorphous solids. Phys. Rev. B 34, 5684-5690 (1986).

4. Anderson, P. W., Halperin, B. I. \& Varma, C. M. Anomalous low-temperature thermal properties of glasses and spin glasses. Philos. Magazine 25, 1-9 (1972).

5. Phillips, W. A. Tunneling states in amorphous solids. J. Low Temp. Phys. 7, 351 (1972).

6. Burin, A. \& Kagan, Y. On the nature of the universal properties of amorphous solids. Phys. Lett. A 215, 191-196 (1996).

7. Lubchenko, V. \& Wolynes, P. G. The microscopic quantum theory of low temperature amorphous solids. Adv. Chem. Phys. 136, 95-206 (2007).

8. Carruzzo, H. M. \& Yu, C. C. Why phonon scattering in glasses is universally small at low temperatures. Phys. Rev. Lett. 124, 075902 (2020).

9. Schechter, M. \& Stamp, P. C. E. Inversion symmetric two-level systems and the low-temperature universality in disordered solids. Phys. Rev. B 88, 174202 (2013).

10. Karpov, V., Klinger, I. \& Ignat'Ev, F. Theory of the low-temperature anomalies in the thermal properties of amorphous structures. Zh. eksp. teor. Fiz 84, 760-775 (1983).

11. Buchenau, U., Galperin, Y. M., Gurevich, V. L. \& Schober, H. R. Anharmonic potentials and vibrational localization in glasses. Phys. Rev. B 43, 5039-5045 (1991).

12. Wallraff, A. et al. Strong coupling of a single photon to a superconducting qubit using circuit quantum electrodynamics. Nature 431, 162 (2004).

13. Koch, J. et al. Charge-insensitive qubit design derived from the cooper pair box. Phys. Rev. A 76, 042319 (2007).

14. Wang, C. et al. Surface participation and dielectric loss in superconducting qubits. Appl. Phys. Lett. 107, 162601 (2015).

15. Lin, Y.-H. et al. Demonstration of protection of a superconducting qubit from energy decay. Phys. Rev. Lett. 120, 150503 (2018).

16. Earnest, $N$. et al. Realization of a $\lambda$ system with metastable states of a capacitively shunted fluxonium. Phys. Rev. Lett. 120, 150504 (2018)

17. Nersisyan, A. et al. Manufacturing low dissipation superconducting quantum processors. in 2019 IEEE International Electron Devices Meeting (IEDM), 31.1.1-31.1.4 (2019)

18. P. M. Place, A. et al. New material platform for superconducting transmon qubits with coherence times exceeding 0.3 milliseconds. arXiv preprint arXiv:2003.00024 (2020).

19. Otterbach, J. et al. Unsupervised machine learning on a hybrid quantum computer. arXiv preprint arXiv:1712.05771 (2017)

20. Amico, M., Saleem, Z. H. \& Kumph, M. Experimental study of shor's factoring algorithm using the ibm q experience. Phys. Rev. A 100, 012305 (2019).

21. Arute, F. et al. Quantum supremacy using a programmable superconducting processor. Nature 574, 505-510 (2019).

22. Müller, C., Cole, J. H. \& Lisenfeld, J. Towards understanding two-level-systems in amorphous solids: insights from quantum circuits. Rep. Prog. Phys. 82, 124501 (2019).

23. Lisenfeld, J. et al. Measuring the temperature dependence of individual two-level systems by direct coherent control. Phys. Rev. Lett. 105, 230504 (2010).

24. Bilmes, A. et al. Electronic decoherence of two-level systems in a josephson junction. Phys. Rev. B 96, 064504 (2017).

25. Barends, R. et al. Coherent josephson qubit suitable for scalable quantum integrated circuits. Phys. Rev. Lett. 111, 080502 (2013).

26. Simmonds, R. W., Lang, K. M., Hite, D. A., Pappas, D. P. \& Martinis, J. M. Decoherence in Josephson qubits from junction resonances. Phys. Rev. Lett. 93, 077003 (2004).

27. Burnett, J. et al. Evidence for interacting two-level systems from the $1 / \mathrm{f}$ noise of a superconducting resonator. Nat. Commun. 5, 4119 (2014).

28. Schlör, S. et al. Correlating decoherence in transmon qubits: low frequency noise by single fluctuators. Phys. Rev. Lett. 123, 190502 (2019).

29. Faoro, L. \& loffe, L. B. Internal loss of superconducting resonators induced by interacting two-level systems. Phys. Rev. Lett. 109, 157005 (2012).

30. Klimov, P. et al. Fluctuations of energy-relaxation times in superconducting qubits. Phys. Rev. Lett. 121, 090502 (2018)

31. Burnett, J. J. et al. Decoherence benchmarking of superconducting qubits. $n p j$ Quant. Inform. 5, 54 (2019).
32. Müller, C., Lisenfeld, J., Shnirman, A. \& Poletto, S. Interacting two-level defects as sources of fluctuating high-frequency noise in superconducting circuits. Phys. Rev. B 92, 035442 (2015).

33. Cooper, K. B. et al. Observation of quantum oscillations between a josephson phase qubit and a microscopic resonator using fast readout. Phys. Rev. Lett. 93 180401 (2004).

34. Neeley, M. et al. Process tomography of quantum memory in a josephson-phase qubit coupled to a two-level state. Nat. Phys. 4, 523 (2008).

35. Shalibo, Y. et al. Lifetime and coherence of two-level defects in a Josephson junction. Phys. Rev. Lett. 105, 177001 (2010).

36. Lisenfeld, J. et al. Decoherence spectroscopy with individual tls. Sci. Rep. 6, 23786 (2016).

37. Meißner, S. M., Seiler, A., Lisenfeld, J., Ustinov, A. V. \& Weiss, G. Probing individual tunneling fluctuators with coherently controlled tunneling systems. Phys. Rev. B 97, 180505 (2018).

38. Grabovskij, G. J., Peichl, T., Lisenfeld, J., Weiss, G. \& Ustinov, A. V. Strain tuning of individual atomic tunneling systems detected by a superconducting qubit. Science 338, 232 (2012).

39. Sarabi, B., Ramanayaka, A. N., Burin, A. L., Wellstood, F. C. \& Osborn, K. D. Projected dipole moments of individual two-level defects extracted using circuit quantum electrodynamics. Phys. Rev. Lett. 116, 167002 (2016).

40. Lisenfeld, J. et al. Electric field spectroscopy of material defects in transmon qubits. npj Quant. Inform. 5, 1-6 (2019).

41. Bilmes, A. et al. Resolving the positions of defects in superconducting quantum bits. Sci. Rep. 10, 1-6 (2020)

42. Martinis, J. M. et al. Decoherence in Josephson qubits from dielectric loss. Phys. Rev. Lett. 95, 210503 (2005).

43. Lisenfeld, J. et al. Observation of directly interacting coherent two-level systems in an amorphous material. Nat. Commun. 6, 6182 (2015).

44. Bilmes, A. Resolving locations of defects in superconducting transmon qubits Dissertation. https://doi.org/10.5445/KSP/1000097557. (2019)

45. Pappas, D. P., Vissers, M. R., Wisbey, D. S., Kline, J. S. \& Gao, J. Two level system loss in superconducting microwave resonators. IEEE Trans. Appl. Superconduct. 21 871-874 (2011).

46. Deng, C., Otto, M. \& Lupascu, A. Characterization of low-temperature microwave loss of thin aluminum oxide formed by plasma oxidation. Appl. Phys. Lett. 104 043506 (2014).

47. Brehm, J. D., Bilmes, A., Weiss, G., Ustinov, A. V. \& Lisenfeld, J. Transmission-line resonators for the study of individual two-level tunneling systems. Appl. Phys. Lett. 111, 112601 (2017).

48. Gao, J. The physics of superconducting microwave resonators. Phd thesis, (2008).

49. Quintana, C. et al. Characterization and reduction of microfabrication-induced decoherence in superconducting quantum circuits. Appl. Phys. Lett. 105, 062601 (2014).

50. Osman, A. et al. Simplified josephson-junction fabrication process for reproducibly high-performance superconducting qubits. arXiv preprint arXiv:2011.05230 (2020).

51. Kang, X. et al. Measurements of tunneling barrier thicknesses for $\mathrm{nb} / \mathrm{al}-\mathrm{alox} / \mathrm{nb}$ tunnel junctions. Phys. C Superconduct. Appl. 503, 29-32 (2014).

52. Zeng, L. J. et al. Direct observation of the thickness distribution of ultra thin AlOxbarriers in al/AlOx/al josephson junctions. J. Phys. D. 48, 395308 (2015)

53. Fritz, S., Radtke, L., Schneider, R., Weides, M. \& Gerthsen, D. Optimization of al/ alox/al-layer systems for josephson junctions from a microstructure point of view. J. Appl. Phys. 125, 165301 (2019).

54. Molina-Ruiz, M. et al. Origin of mechanical and dielectric losses from two-level systems in amorphous silicon. arXiv preprint arXiv:2008.07489v1 (2020).

55. Grünhaupt, L. et al. An argon ion beam milling process for native alox layers enabling coherent superconducting contacts. Appl. Phys. Lett. 111, 072601 (2017).

\section{ACKNOWLEDGEMENTS}

A.B. acknowledges support from the Helmholtz International Research School for Teratronics (HIRST) and the Landesgraduiertenförderung-Karlsruhe (LGF). J.B. was financially supported by Studienstiftung des Deutschen Volkes. J.L. gratefully acknowledges funding from the Deutsche Forschungsgemeinschaft (DFG), grant LI2446-1/2. A.V.U acknowledges support provided by the Ministry of Education and Science of the Russian Federation in the framework of the Program to Increase Competitiveness of the NUST MISIS (contract No. K2-2020-017). The work was also supported by the Initiative and Networking Fund of the Helmholtz Association and by Google LLC. We acknowledge support by the KIT-Publication Fund of the Karlsruhe Institute of Technology. We acknowledge Johannes Rotzinger and loan Pop for fruitful discussions, Silvia Diewald and Patrice Brenner for their technical assistance with electron-beam devices, and Lucas Radtke for his indispensable assistance in the clean-room. 


\section{AUTHOR CONTRIBUTIONS}

The qubit samples were designed and fabricated by A.B. Experiments were devised and performed by J.L. in a setup implemented by A.B. and J.L. S.V. performed calculations for the mutually coupled TLS system, and J.B. simulated the electric-field distribution of nanogap capacitors. The manuscript was written by J.L. and A.B. with contributions from all authors.

\section{FUNDING}

Open Access funding enabled and organized by Projekt DEAL.

\section{COMPETING INTERESTS}

The authors declare no competing interests.

\section{ADDITIONAL INFORMATION}

Supplementary information is available for this paper at https://doi.org/10.1038/ s41534-020-00359-x.

Correspondence and requests for materials should be addressed to A.B.
Reprints and permission information is available at http://www.nature.com/ reprints

Publisher's note Springer Nature remains neutral with regard to jurisdictional claims in published maps and institutional affiliations.

cc) (i)

Open Access This article is licensed under a Creative Commons Attribution 4.0 International License, which permits use, sharing, adaptation, distribution and reproduction in any medium or format, as long as you give appropriate credit to the original author(s) and the source, provide a link to the Creative Commons license, and indicate if changes were made. The images or other third party material in this article are included in the article's Creative Commons license, unless indicated otherwise in a credit line to the material. If material is not included in the article's Creative Commons license and your intended use is not permitted by statutory regulation or exceeds the permitted use, you will need to obtain permission directly from the copyright holder. To view a copy of this license, visit http://creativecommons. org/licenses/by/4.0/.

(c) The Author(s) 2021 